\title{
Research on terrain aerodynamics analysis of power grid structure at different temperature
}

\author{
$Y u \mathrm{Ye}^{1,2, *}$, Xunjian $\mathrm{Xu}^{1,2}, L i$ Yang, and Yusheng $\mathrm{Yi}^{1,2}$ \\ ${ }^{1}$ State Key Laboratory of Disaster Prevention \& Reduction for Power Grid Transmission and \\ Distribution Equipment, Changsha, China \\ ${ }^{2}$ State Key Hunan Electric Power Company Disaster Prevention and Reduction Center, Changsha, \\ China
}

\begin{abstract}
The distribution of near-surface meteorological elements will be greatly affected by topography and other factors, such as wind, which making the dynamic structure of the micro-topography area passed by the power transmission and transformation equipment more susceptible to micro-topography climate. The single hill is one of the typical topography in the micro-topography. This paper studies the variation of buoyant flow and temperate circulation in a typical long hilly terrain. Integrating factors such as buoyancy, turbulence and micro-topography, the separation vortex simulation technology is adopted to the special conditions of microtopography climate. The effect of different surface temperatures on the wind field of the power grid is described in detail. Experiments in the thesis show that the difference in surface temperature will directly affect the speedup ratio. Compared with the change of air temperature, when the surface temperature is higher, the acceleration effect is less obvious, but when the surface temperature is lower, the acceleration effect is more obvious.
\end{abstract}

Keywords: Aerodynamics; Wind field simulation; Micro terrain area; Grid structure.

\section{Background}

Due to the microclimate caused by topography, the transmission lines in the microtopography area are prone to icing. A simple case of how terrain affects local weather is the alleged narrowing effect, which accelerates when the wind passes through a narrow duct. Unfortunately, when complex or high-resolution terrain is considered in the dynamics framework of numerical weather prediction models(Zhou B [1], Wyngaard [2]), there are still many difficulties and challenges in providing higher-resolution wind fields. Recently the simulation of computational fluid dynamics (CFD) dealing with the complex microclimate process in the micro-topography area has attracted more and more attention. According to the various methods of solving the NS equations, there are currently two recognized CFD simulation methods, namely the Reynolds average NS equation (RANS) method (Castellani F[3]) and the large eddy simulation (LES) method (Hu P[4]).

\footnotetext{
* Corresponding author: yuree2008@126.com
} 
The research of mountain wind field simulation began in the 1980s, Taylor sorted out this and compared it with theoretical calculations. It can be considered that the measurement result near the surface is far from the theoretical calculation result of the acceleration ratio. On the basis of numerical simulation, Song D[5] sorted out and compared the wind tunnel test of two-dimensional steep and the numerical simulation of gentle slopes. The above-mentioned research has two shortcomings: First, the most standard models come from Fluent, and does not consider the buoyancy and other factors. Second, the existing literature rarely considers the effect of temperature on airflow. In mountain transmission lines, due to the large temperature difference between the mountain and the air, flashover accidents and galloping accidents are prone to occur in strong winds. We chose the CFD tool OpenFOAM (Modesti D[6], Weller H[7], Zhu[8]) as the development platform tool, considering the effects of temperature and buoyancy.

In the paper, the aerodynamic characteristics of terrain and roughness at different temperatures are studied numerically. Firstly, we briefly describe the methods used to handling physical problems in numerical processing in Section 1. The boundary and initial conditions of the governing equation are discussed in Section 2. Followed by Section 3 discusses the characteristics of meteorological elements in typical hilly areas under several different temperature conditions and rough surfaces. Finally, the aerodynamic characteristics and result analysis of different temperature models are discussed, and a summary conclusion is given in Section 4.

\section{Problem description and physical model}

As we all know, the common research types of atmospheric circulation include two processes: backflow or acceleration areas caused by structural obstacles or microtopographic areas. The other is the buoyancy effect, which is mainly caused by the flow of heat from the surface or the direction of the surface. Important vertical airflow acceleration is bound to occur. However, as a typical fluid dynamics simulation tool, OpenFOAM has not been used to study typical valleys to neutralize the results of temperature changes and buoyant flow caused by circulation.

\subsection{Mathematical physics equations and OpenFOAM realization}

For simplicity, we used a quasi-compressible approximation with density as an explicit variable. Oliveira [9] introduced the pressure and velocity coupling algorithm in OpenFOAM in detail. Considering the impact of compressibility, the filtered version of the general continuity equation was adopted by us (for simplicity, the bar graph above large variables is not selected): in our case, we used a quasi-compressible approximation, The density is used as an explicit variable. Oliveira[9] introduced the pressure and velocity coupling algorithm in OpenFOAM in detail.

$$
\frac{\partial \rho}{\partial t}+\nabla \cdot(\rho \vec{U})=0
$$

The momentum control equation is:

$$
\frac{\partial}{\partial t}\left(\rho u_{i}\right)+\frac{\partial}{\partial x}\left(\rho u_{i} u_{j}\right)-\nabla \cdot\left(T+T_{s g s}\right)=-\nabla p+\rho \vec{g}-\nabla p^{*}+f_{c}
$$

here $T$ indicates the stress tensor related to molecular viscosity. 


$$
T=\mu\left[\left(\frac{\partial u_{i}}{\partial x_{j}}+\frac{\partial u_{j}}{\partial x_{i}}\right)-\frac{2}{3}\left(\frac{\partial u_{k}}{\partial x_{i}}\right) \delta_{i j}\right]
$$

Among them $T_{s g s}$ is the stress tensor at the sub-grid scale. OpenFOAM can provide different options. Among them, $\nabla p^{*}$ in (2) corresponding to the external force is used to the maintain flow. Although we choose coriolis $\left(\vec{f}_{c}\right)$ in (2), but in our calculations In the example, this force is very weak, so you don't need to consider it. The advanced nature of choosing OpenFOAM lies in each application by numerical calculation can be discrete for the parameters. The combined equation of temperature and enthalpy equation is:

$$
\frac{\partial \rho h}{\partial t}+\nabla \cdot(\rho \vec{U} h)+\frac{\partial \rho K}{\partial t}+\nabla \cdot(\rho \vec{U} K)-\nabla \cdot\left(\alpha_{t} \nabla h\right)-r a d=\frac{D p}{D t}
$$

here $h$ represents the specific enthalpy, $K=\lfloor\vec{U}\rfloor / 2$ represents the mechanical energy. In the equation (4), the sum of the molecular thermal diffusivity can be described the effective thermal diffusivity $\alpha_{t}$ of SGS. The radiative transmission in the diffusion coefficient formula (4) takes into account the use of the radiation model feasible in OpenFOAM (Vdovin $\mathrm{A}[10,11])$. Generally speaking, in our case, there is no active radiation. Use the ideal gas equation of state

$$
p=\rho R T
$$

where $\mathrm{R}=287 \mathrm{~J} /(\mathrm{Kg}$. K). To embody passive temperature in the transfer model, we choose

$$
\frac{\partial \rho T}{\partial t}+\nabla \cdot(\rho \vec{U} T)-\nabla \cdot\left(\alpha_{t} \nabla T\right)=f_{v}
$$

here $T$ represents the temperature, $f_{v}$ is the heat source. In this paper, it is the temperature. This feature is part of the process is allowed (Melvin[12]).

In short, the above-mentioned solvers for wind field simulation mainly include the following equations: Navier-Stokes, continuity, temperature equations and enthalpy conservation, these equations can be solved using tools and libraries which provided by the useful platform OpenFOAM.

\subsection{Initial boundary conditions}

In general, although several predefined conditions will be provided by OpenFOAM. Before each simulation is applied, it is important to create specific conditions. When we use DES for buoyancy simulation, these conditions will be described in detail.

The logarithmic boundary conditions commonly used in atmospheric sciences are adopted, i.e. $U(z)=\frac{U_{*}}{\kappa} \ln \left(\frac{z-z_{\text {Ground }}+z_{0}}{z_{0}}\right), k=\frac{U_{*}^{2}}{\sqrt{C_{\mu}}}, \varepsilon=\frac{U_{*}^{3}}{\kappa\left(z+z_{0}\right)}$, here $\kappa$ (von 
Karmans constant), $U_{*}$ (friction speed), $\mathrm{z}_{0}$ (surface roughness length) and $z_{\text {Ground }}$ (displacement height) can be estimated from the data or be known. $\kappa$ and $\varepsilon$ are satisfied the conservation equation $\sigma_{\varepsilon}=\frac{\kappa^{2}}{\left(c_{\varepsilon 2}-c_{\varepsilon 1}\right) \sqrt{c_{\mu}}}$, here $\sigma_{\varepsilon}, c_{\varepsilon 2}, c_{\varepsilon 1}, c_{\mu}$ are the coefficient of the model $k-\varepsilon$. For large airflow, we employ the roughness length $\mathrm{z}_{0}$. The $\mathrm{z}_{0}, k_{s}$ can be estimated by $z_{0}=20 k_{s}$, here $k_{s}$ wall function does not choose the first element center at a certain distance from the wall $k_{s}$. For low ones, the crop border $\mathrm{z}_{0} \approx 0$ and $k_{s}=40 \mathrm{~m}$.

\section{Calculation results and discussion}

We will discuss the buoyant airflow on the hilly terrain where the grid structure is located under several different temperature conditions in this section .

This paper mainly studies the neutral layered boundary layer. Based on the experimental data of three-dimensional rough surface hills by W.Lou and Huai. The important feature of hilly terrain is that it has a cosine-squared cross-section, as shown in Fig.1, which can be expressed as

$$
z_{s}=\left\{\begin{array}{c}
h \cos ^{2}\left(\pi \frac{r}{2 L}\right), r<L \\
0, r \geq L
\end{array}\right.
$$

The height of the mountain is $100 \mathrm{~m}$, the radius of the bottom surface is $150 \mathrm{~m}$, and the slope is stable. The size of the computational domain is. Considering our interest in dealing with irregular meshing, snappyHexMesh is the preferred strategy to solve the mesh generation problem.

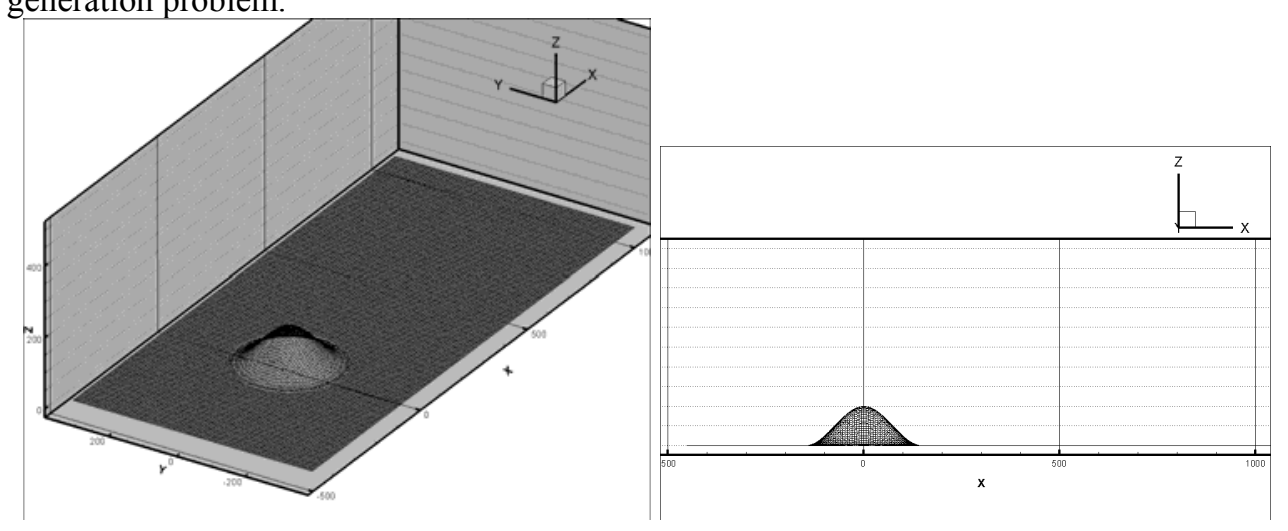

Fig. 1. Cosine Mountain model meshing.

Among them, laminar flow viscosity, thermal expansion coefficient $\beta=3 \mathrm{e}-3$, laminar flow Prandtl number $\mathrm{P}_{\mathrm{r}}=0.7$, reference temperature $\mathrm{T}_{\text {Ref }}=300$, turbulent parameter $\mathrm{P}_{\mathrm{rt}}=0.85$. It is easy to see that the temperature parameters used in the numerical simulation are summarized in Table 1, where $Z_{\text {ref }}=10$ and $U_{\text {ref }}=10.0$. A series of simulation experiments were instructed for different surface temperatures (see Table 1). For the cases that are not 
in Table 1, that is, the effects of atmospheric buoyancy and temperature phase in the traditional algorithm are not considered.

Table 1. Boundary condition setting under different temperature conditions.

\begin{tabular}{|c|c|c|c|c|c|}
\hline Case & Inlet/Outlet & InternalField & Ground & Terrain & $\begin{array}{c}\text { Top And } \\
\text { Sides }\end{array}$ \\
\hline T0 & \multirow{4}{*}{ zeroGradient } & \multirow{4}{*}{$\begin{array}{l}\text { uniform by } \\
283.15\end{array}$} & $\begin{array}{c}273.15 \mathrm{~K} \\
\text { fixedValue; }\end{array}$ & $\begin{array}{c}273.15 \mathrm{~K} \\
\text { fixedValue; }\end{array}$ & \multirow{4}{*}{ symmetry } \\
\hline $\mathrm{T} 10$ & & & $\begin{array}{c}283.15 \mathrm{~K} \\
\text { fixedValue; }\end{array}$ & $\begin{array}{c}283.15 \mathrm{~K} \\
\text { fixedValue; }\end{array}$ & \\
\hline $\mathrm{T} 20$ & & & $\begin{array}{c}293.15 \mathrm{~K} \\
\text { fixedValue; }\end{array}$ & $\begin{array}{c}293.15 \mathrm{~K} \\
\text { fixedValue; }\end{array}$ & \\
\hline $\mathrm{T} 30$ & & & $\begin{array}{c}303.15 \mathrm{~K} \\
\text { fixedValue; }\end{array}$ & $\begin{array}{c}303.15 \mathrm{~K} \\
\text { fixedValue; }\end{array}$ & \\
\hline
\end{tabular}

The single mountain model studied here adopts the cosine mountain model of formula (7). The shape of the mountain is the same as that of the length mountain, as shown in Fig.1.

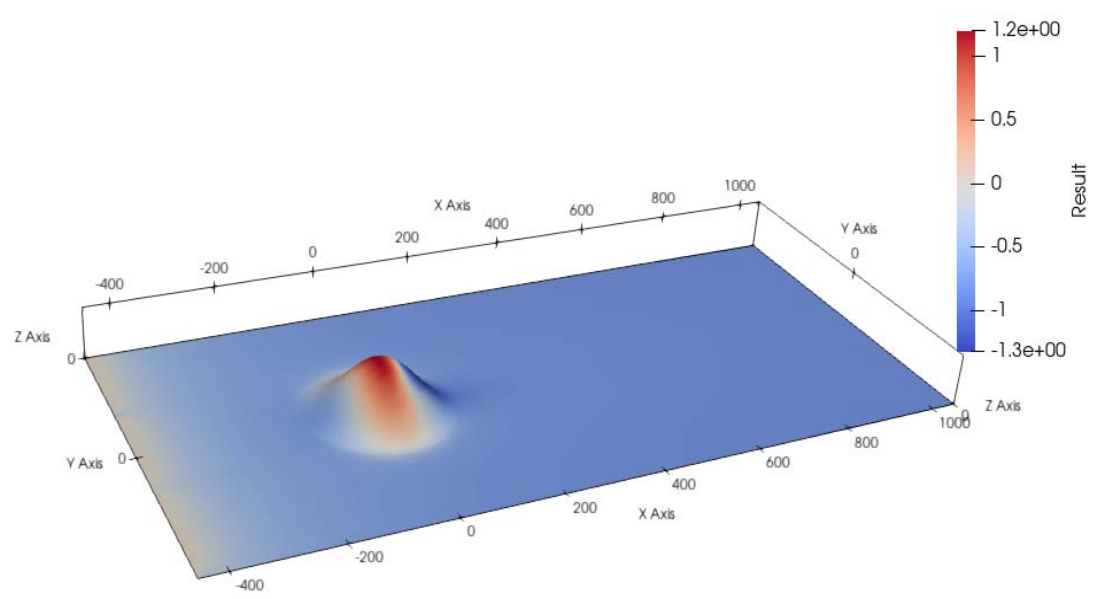

Fig. 2. Regardless of temperature, the acceleration ratio distribution map at a height of $10 \mathrm{M}$ above the ground (mountain).

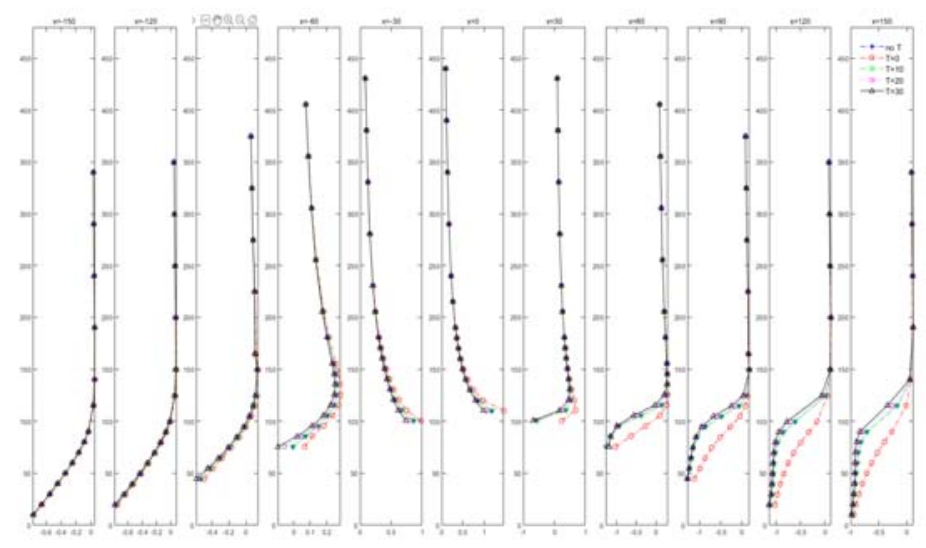

Fig. 3. Acceleration ratio of wind speed on off-wind surface at different $X$ positions at different temperatures at the top of the mountain $(\mathrm{Y}=0)$. 
The experimental results are shown in Figure 2 to Figure 3. From the figure, we can draw:

1) For different temperatures, the maximum acceleration ratio appears at the top of the mountain at the $\mathrm{Y}=0$ plane position.

2) Under different temperature conditions, the position with the largest acceleration ratio appears at the top side of the windward, and the acceleration effect is the most obvious.

3) The acceleration ratio decreases with the increase of the distance from the ground (mountain) surface and approaches 0 , and the influence of the wake area in the height direction is weakened.

4) There is a small deceleration zone in front of the mountain, and the downhill section of the mountain is the deceleration zone;

5) When there is no temperature difference between the mountain temperature and the air temperature, the conclusions of working condition T10 and working condition NoT are almost the same. It also proves that the algorithm used in this project is correct.

6) When the surface temperature of the mountain is higher than the air temperature, the shorter the length of the affected area of the mountain acceleration area, the less obvious the acceleration effect.

\section{Discussion and conclusion}

This paper mainly studies the dynamic down scaling model and parametric wind field model under basic aerodynamic information, mainly to improve the calculation accuracy, and its conclusions are as follows:

(1) The surface temperature under the grid structure affects the wind speed ratio above the surface. When the temperature in the hilly area is lower than the surface temperature, the acceleration effect of the wind field on the surface of the hilly terrain is most distinctest. The acceleration rate is the best. When the air temperature is lower than the temperature in the hilly area, for a long-length hilly terrain, the acceleration effect will be weakened. The lower the temperature, the longer the acceleration zone.

(2) When the grid structure is in the peak computing environment, at the top of the windward surface, the maximum wind speed ratio above the hilly terrain will reach its maximum value. The wind acceleration effect is very closely related to the hilly terrain. Once close to the hilly terrain, the wind acceleration effect increases; at the same time, as the hilly terrain becomes lower, the wind acceleration effect becomes greater. Therefore, the wind around the top of the hilly terrain has a significant acceleration effect.

This research only reveals the dynamic change characteristics of wind speed under different temperature conditions in typical mountainous terrain under the power grid structure. How to consider the influence of wind speed in state grid equipment inspection and engineering design is still to be further studied.

\section{Acknowledgements}

Project supported by the State Grid Science and Technology of China (Grant No. 5216A019007J) Research on the prediction technology of micro-terrain regional power grid galloping, Research on the prediction and application technology of winter ice wind temperature near the ground in the micro-topography of the power grid. 


\section{References}

1. Zhou B, Simon J S, Chow F K. The convective boundary layer in the terra incognita[J]. Journal of the Atmospheric Sciences, 2014, 71(7): 2545-2563.

2. Wyngaard J C. Toward numerical modeling in the "Terra Incognita" $[\mathrm{J}]$. Journal of Atmospheric Sciences, 2004, 61(14): 1816-1826.

3. Castellani F, Astolfi D, Mana M, et al. Investigation of terrain and wake effects on the performance of wind farms in complex terrain using numerical and experimental data[J]. Wind Energy, 2017, 20(7): 1277-1289.

4. Hu P, Li Y, Han Y, et al. Numerical simulations of the mean wind speeds and turbulence intensities over simplified gorges using the SST k- $\omega$ turbulence model[J]. Engineering Applications of Computational Fluid Mechanics, 2016, 10(1): 359-372.

5. Song D, Yang J, Cai Z, et al. Wind estimation with a non-standard extended Kalman filter and its application on maximum power extraction for variable speed wind turbines[J]. Applied energy, 2017, 190: 670-685.

6. Modesti D, Pirozzoli S. A low-dissipative solver for turbulent compressible flows on unstructured meshes, with OpenFOAM implementation[J]. Computers \& Fluids, 2017, 152: $14-23$.

7. Weller H, Tabor G, Jasak H, Fureby C. A tensorial approach to computational continuum mechanics using object-oriented techniques. Comput Phys 1998;12(6):62031 .

8. Zhu L, Chen S, Guo Z. dugksFoam: An open source OpenFOAM solver for the Boltzmann model equation[J]. Computer Physics Communications, 2017, 213: 155164.

9. Oliveira P J, Steffen J L, Cheung P. Parameter estimation of seasonal ARIMA models for water demand forecasting using the Harmony Search Algorithm[J]. Procedia Engineering, 2017, 186: 177-185.

10. Vdovin A, Gustafsson M, Sebben S. A coupled approach for vehicle brake cooling performance simulations[J]. International Journal of Thermal Sciences, 2018, 132: 257-266.

11. Vdovin A. Radiation heat transfer in Openfoam. Final Assignment for the Course CFD with OpenSource Software; 2009.

12. Melvin J, Rao P, Kaufman R, et al. Atomic scale mixing for inertial confinement fusion associated hydro instabilities[J]. High Energy Density Physics, 2013, 9(2): 288296. 